\title{
Altered intrinsic brain activity and regional cerebral blood flow in patients with chronic neck and shoulder pain
}

\author{
Xi Yue ${ }^{C, D, E}$, Yong Du ${ }^{A}$ \\ Department of Radiology, Affiliated Hospital of North Sichuan Medical College, Nanchong, China
}

\section{Abstract}

Purpose: To identify the changes of intrinsic brain activity and regional cerebral blood flow in patients with chronic neck and shoulder pain (CNSP) by using amplitude of low-frequency fluctuation (ALFF) analysis and arterial spin labelling study.

\begin{abstract}
Material and methods: In total, 28 CNSP patients and 25 age-matched and sex-matched healthy controls (HCs) participated in the study. Resting-state functional magnetic resonance imaging (rs-fMRI) and arterial spin labelling (ASL) MRI were acquired. Correlations between ALFF and cerebral blood flow (CBF) were analysed. Subsequently, the differences in ALFF and CBF were compared in the two groups. Finally, the visual analogue scale (VAS) was also assessed in the CNSP group.

Results: Compared with HCs, CNSP patients showed significantly abnormal ALFF and CBF in several brain regions, including the cerebellum posterior lobe, middle orbitofrontal gyrus, medial superior frontal gyrus, middle temporal gyrus, precuneus, cingulate gyrus, middle occipital gyrus, middle frontal gyrus, postcentral gyrus, precentral gyrus, and superior parietal gyrus. Correlation analysis showed that the ALFF value of the medial superior frontal gyrus positively correlated with the VAS score. However, no correlation was found between the CBF values and the VAS score.

Conclusions: The altered ALFF and CBF values in CNSP patients were observed in different pain-related brain regions that were involved in pain modulation and perception. The combination of rs-fMRI and ASL MRI might provide complementary information for increasing our understanding of the neuropathology in CNSP.
\end{abstract}

Key words: chronic neck and shoulder pain, cervical spondylotic radiculopathy, resting-state functional magnetic resonance imaging, amplitude of low-frequency fluctuation, arterial spin labelling, cerebral blood flow.

\section{Introduction}

Cervical spondylotic radiculopathy (CSR) is the most common pattern of cervical spondylosis, which is a common degenerative disease [1]. Chronic neck and shoulder pain (CNSP) is a common clinical symptom of CSR [2]. It is estimated that over $20 \%$ of the population in Germany suffer from CNSP [3]. Many studies have investigated the underlying cerebral abnormalities caused by chronic pain, including changes in brain structure, function, chemistry, and blood flow [4-8]. A few brain imaging studies have shown that CNSP can cause changes of spontaneous neural activities $[9,10]$.

Resting-state functional magnetic resonance imaging (rs-fMRI), widely used in chronic pain diseases [11-15], is an effective tool to evaluate the intrinsic neural activity of the human brain $[16,17]$. The rs-fMRI analysis methods mainly include amplitude of low-frequency fluctuation (ALFF), regional homogeneity (ReHo) [9], and functional connectivity (FC) [10]. ALFF is calculated to measure the low-frequency $(0.01 \sim 0.08 \mathrm{~Hz})$ spontaneous blood-oxygen-level-dependent (BOLD) fluctuations that

Correspondence address:

Yong Du, Department of Radiology, Affiliated Hospital of North Sichuan Medical College, Wenhua Road No. 63, Nanchong 637000, Sichuan province, China,

phone: +86-817-2262089, e-mail:dy_nsmc@163.com

Authors' contribution:

A Study design · B Data collection · C Statistical analysis · D Data interpretation · E Manuscript preparation · F Literature search · G Funds collection 
are meaningful and are related to neural spontaneous activity [18]. The advantage of this method is that it can detect the corresponding region of impairment, which may prove to be a therapeutic target for CNSP patients in the future.

Cerebral blood flow (CBF) is closely related to brain metabolism, such as oxygen consumption, which can influence the BOLD signal [19]. Hence, ALFF does not completely explain the pathophysiological mechanism of pain. Recent studies have explored the relationship between CBF and rs-fMRI metrics [20, 21]. Arterial spinlabelled (ASL) perfusion MRI permits noninvasive quantification of CBF, which can be grouped into three types: continuous labelling, pulsed labelling, and velocity selective labelling [22]. This technique has been used to explore $\mathrm{CBF}$ alterations in patients with cervical spondylotic myelopathy and has found increased CBF in operculum-integrated and motor control regions, and decreased CBF in sensory and motor-sensory processing regions [23]. ASL MRI therefore may provide complementary information for understanding the pathophysiology of CNSP.

To our knowledge, very few studies have simultaneously explored alterations of intrinsic brain activity and CBF in patients with CNSP. Therefore, in this study, we combined two MRI techniques, rs-fMRI and ASL, to systematically investigate alterations of ALFF and CBF in whole brain and possible relationships between ALFF and CBF. We also correlated the altered values of ALFF and CBF in CNSP patients to some clinical data. Therefore, we hypothesized that CNSP patients will present with aberrant regions of ALFF and CBF values, which might be involved in pain modulation and could become therapeutic targets to help clinicians relieve pain and improve the quality of life of CNSP patients.

\section{Material and methods}

\section{Participants}

From January 2019 to October 2019, a total of 53 righthanded individuals were recruited for this study, including 28 CNSP patients and $25 \mathrm{HCs}$. This study was approved by the Ethics Committee of the Affiliated Hospital of North Sichuan Medical College, and all the subjects voluntarily signed a informed consent form before this study.

The CNSP group met the following criteria: (1) a history of neck and shoulder pain for more than three months; (2) cervical disc herniation and nerve root compression diagnosed by conventional MRI; (3) no pain in other parts of body; and (4) did not receive any pain treatment before the study. Exclusion criteria for all subjects were as follows: (1) neck and shoulder pain caused by other reasons, such as periarthritis of shoulder; (2) structural abnormalities of the brain on conventional MRI, such as tumour, cerebral infarction, and obvious cerebral atrophy; and (3) psychiat$\mathrm{ric} /$ neurological diseases affecting cognition.
The demographic data were recorded before the MRI scan. In order to find out some biomarkers that can predict CNSP, we also collected some clinical data, including disease duration (the time between the diagnosis and the experiment), and visual analogue scale (VAS) scores to assess the pain intensity.

\section{Image acquisition}

MRI data were acquired using a 3.0 Tesla scanner (Discovery MR750, General Electric Company) with a 32-channel head coil in our hospital. We fixed the head with a foam pad and reduced the influence of external sound with earplugs. During the scan, subjects were required to relax, close their eyes, remain awake, and think about nothing. All scan operations were performed by one skilled MRI radiologist.

The parameters of three-dimensional T1-weighted imaging (3D-T1WI) were as follows: repetition time $(\mathrm{TR})=5.5 \mathrm{~ms}$; echo time $(\mathrm{TE})=1.6 \mathrm{~ms}$; slices = 144; flip angle $=9^{\circ}$; field of view $($ FOV $)=240 \mathrm{~mm} \times 240 \mathrm{~mm}$; matrix $=256 \times 256$; and slice thickness $=1 \mathrm{~mm}$.

Rs-fMRI was acquired using an echoplanar image sequence with parameters as follows: $\mathrm{TR}=2000 \mathrm{~ms}$; $\mathrm{TE}=29 \mathrm{~ms}$; slices $=36$; flip angle $=90^{\circ} ; \mathrm{FOV}=240 \mathrm{~mm}$ $\times 240 \mathrm{~mm}$; matrix $=64 \times 64$; and slice thickness $=3 \mathrm{~mm}$.

The parameters of three-dimensional pseudo-continuous ASL (pcASL) sequence were as follows: TR = $5327 \mathrm{~ms}$; $\mathrm{TE}=10.5 \mathrm{~ms}$; slices $=36$; post-label delay= $2525 \mathrm{~ms}$; bandwidth $=976.6 \mathrm{~Hz}$; flip angle $=111^{\circ}$; FOV $=$ $240 \times 240 \mathrm{~mm}$; matrix $=64 \times 64$; and slice thickness $=$ $4 \mathrm{~mm}$.

\section{Amplitude of low-frequency fluctuation preprocessing and analysis}

The rs-fMRI images were preprocessed by using the toolbox for Data Processing \& Analysis of Brain Imaging (DPABI, http://rfmri.org/DPABI) based on MATLAB R2018b. The first 10 time points were removed to make the magnetic field steady and to allow the subjects adapt to the scanning environment. Then, the remaining functional images underwent slice timing to avoid the differences of slice acquisition time and were realigned to the first image for head motion correction. Next, subjects with head movements more than $1.5 \mathrm{~mm}$ in translation and $1.5^{\circ}$ in rotation of any direction were excluded. Then, the corrected images were normalised to the standard Montreal Neurological Institute (MNI) space and were resampled to $3 \mathrm{~mm}$ cubic voxels. Next, the images were smoothed with a $4 \mathrm{~mm}$ full width at half maximum (FWHM) Gaussian kernel to reduce noise. After that, linear detrending and bandpass filtering (from 0.01 to $0.08 \mathrm{~Hz}$ ) were performed to reduce the influence of low-frequency drifts and high-frequency physiological noise [24]. Finally, the white matter 
signal, the cerebrospinal fluid signal, the global signal, and Friston 24 head motions were removed as nuisance covariates.

The ALFF maps were calculated using the DPABI toolbox according to the ALFF method proposed by Zang et al. [25] in 2007. Then, the time series retained by the above band-pass filter were converted from time to frequency domains using the fast Fourier transform (FFT) algorithm and the power spectrum was obtained. Finally, ALFF values of each voxel were normalised by Fisher z-transformation.

\section{Cerebral blood flow preprocessing and analysis}

CBF maps were calculated automatically by a General Electric scanner. The ASL data were preprocessed by using Statistical Parametric Mapping (SPM) 12 (http://www. fil.ion.ucl.ac.uk/spm) based on MATLAB R2018b. First, the T1-weighted structural images were segmented into grey matter, white matter, and cerebrospinal fluid, which were subsequently resliced to the ASL image space. Then the CBF images were written into the MNI space using the deformation parameter derived from the prior step and were resliced into a $3 \mathrm{~mm}$ cubic voxel. Finally, each co-registered CBF map was spatially smoothed with a Gaussian kernel of $8 \mathrm{~mm}$ FWHM.

\section{Statistical analysis}

The demographics and clinical data between the two groups were compared by SPSS 23.0. Two-sample $t$ tests were used to detect the differences in the age, education, VAS scores, and pain duration between CNSP patients and HCs. The $\chi^{2}$ test was used to examine the difference in sex composition between the two groups. The statistical significance level was set at a threshold of $p<0.05$.

The group analysis was performed using two-sample $t$ tests in the toolbox of DPABI software to assess the differences of ALFF and CBF between the two groups with age, gender, and education as the covariates. The results were multiple compared and corrected by AlphaSim. The mean ALFF and CBF values of the altered brain areas were extracted from each patient's ALFF and CBF map by DPABI toolbox. Then, these abnormal values in CNSP patients were correlated to clinical data including VAS scores and pain duration by using Pearson correlation analysis in SPSS 23.0.

\section{Results}

\section{Demographics and clinical data}

The statistical results of demographics and clinical data of the two groups are listed in Table 1. There was no statistically significant difference in demographic variables between the two groups $(p>0.05)$.
Table 1. Demographic and clinical characteristics of subjects

\begin{tabular}{|l|c|c|c|} 
Factor & $\begin{array}{c}\text { CNSP patients } \\
(n=28)\end{array}$ & $\begin{array}{c}\text { HCs } \\
(n=25)\end{array}$ & $p$ value \\
\hline Age (years) & $47.04 \pm 8.74$ & $43.56 \pm 11.96$ & 0.238 \\
\hline Sex (male/female) & $17 / 11$ & $13 / 12$ & 0.718 \\
\hline Education (years) & $10.75 \pm 0.76$ & $12.52 \pm 0.93$ & 0.143 \\
\hline Pain duration (months) & $32.56 \pm 27.72$ & & \\
\hline VAS score & $5.44 \pm 1.71$ & & \\
\hline
\end{tabular}

There were no significant differences in demographic variables between the two groups $(p>0.05)$. CNSP - chronic neck and shoulder pain, HCs - healthy controls

\section{Comparison of amplitude of low-frequency fluctuation between groups}

Compared with the HCs, CNSP patients showed decreased ALFF in the left middle temporal gyrus, left precuneus, left precentral gyrus, right cingulate gyrus, right middle occipital gyrus, right middle frontal gyrus, and right postcentral gyrus, and increased ALFF in the bilateral cerebellum posterior lobe, right precuneus, left middle orbitofrontal gyrus, and left medial superior frontal gyrus (Figure 1 and Table 2).

\section{Comparison of cerebral blood flow between groups}

Compared with HCs, CNSP patients showed significantly decreased $\mathrm{CBF}$ in the left precentral gyrus and right middle frontal gyrus, and increased CBF in the bilateral superior parietal gyrus and right middle temporal gyrus (Figure 2 and Table 3).

\section{Correlations between amplitude of low-frequency fluctuation values and clinical data}

As shown in Figure 3, the ALFF value of the left medial superior frontal gyrus was positively correlated with the VAS score. But there was no significant statistical relationship between the ALFF values and pain duration.

\section{Correlations between cerebral blood flow values and clinical data}

According to the correlation analysis, there was no significant statistical relationship between the CBF values and the VAS score or pain duration.

\section{Discussion}

To our knowledge, this is the first study to investigate alterations in brain function and blood flow in patients with CNSP by combining BOLD and ASL techniques. Compared to HCs, the ALFF values decreased in the left middle temporal gyrus, left precuneus, left precentral gyrus, right cingulate gyrus, right middle occipital gyrus, right middle frontal gyrus, and right postcentral gyrus, 


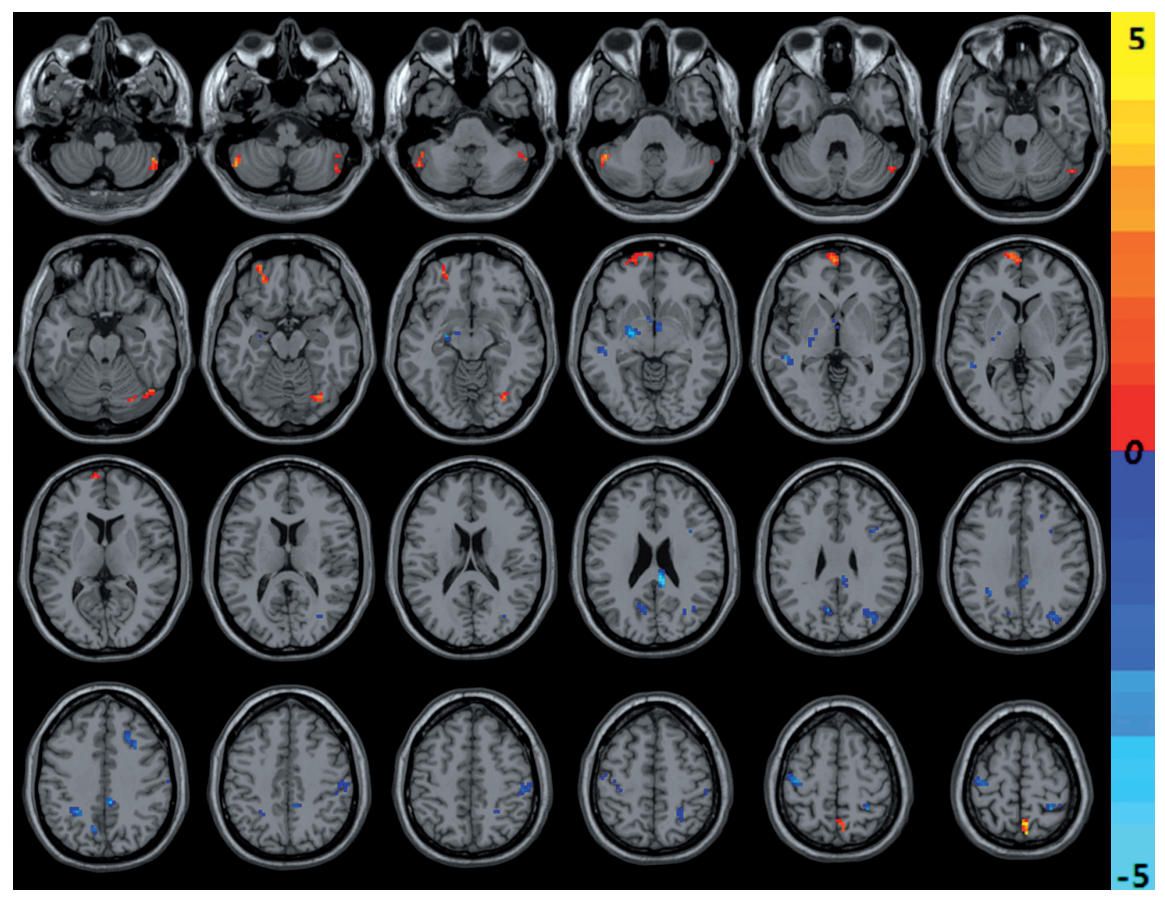

Figure 1. The cold and warm colours, respectively, indicated that the amplitude of low-frequency fluctuation value decreased and increased in the brain regions in chronic neck and shoulder pain patients compared with healthy controls $(p<0.05$, AlphaSim corrected)

Table 2. Brain regions with amplitude of low-frequency fluctuation differences between the chronic neck and shoulder pain and healthy controls groups

\begin{tabular}{|c|c|c|c|c|c|}
\hline \multirow[t]{2}{*}{ Region (R; L) } & \multirow{2}{*}{$\begin{array}{l}\text { Voxel } \\
\text { size }\end{array}$} & \multicolumn{3}{|c|}{ Peak MNI coordinate } & \multirow{2}{*}{$\begin{array}{l}\text { Peak } \\
t \text {-value }\end{array}$} \\
\hline & & $x$ & $Y$ & $z$ & \\
\hline \multicolumn{6}{|l|}{ CNSP $<\mathrm{HCS}$} \\
\hline Temporal_Mid_L & 17 & -51 & -33 & 0 & -3.4468 \\
\hline Precuneus_L & 33 & -9 & -66 & 27 & -4.1183 \\
\hline Precentral_L & 58 & -42 & -18 & 60 & -3.5049 \\
\hline Cingulate Gyrus_R & 51 & 9 & -36 & 24 & -4.4834 \\
\hline Occipital_Mid_R & 38 & 33 & -72 & 33 & -3.0194 \\
\hline Frontal_Mid_R & 20 & 24 & 21 & 36 & -3.5481 \\
\hline Postcentral_R & 32 & 30 & -45 & 60 & -3.4172 \\
\hline \multicolumn{6}{|l|}{$\mathrm{CNSP}>\mathrm{HCS}$} \\
\hline Precuneus_R & 24 & 6 & -60 & 63 & 3.4639 \\
\hline Frontal_Sup_Medial_L & 31 & -3 & 63 & 0 & 2.8734 \\
\hline $\begin{array}{l}\text { Cerebellum posterior } \\
\text { lobe_L }\end{array}$ & 32 & -51 & -57 & -45 & 2.977 \\
\hline $\begin{array}{l}\text { Cerebellum posterior } \\
\text { lobe_R }\end{array}$ & 41 & 39 & -63 & -57 & 3.3063 \\
\hline
\end{tabular}

CNSP - chronic neck and shoulder pain, HCs - healthy controls, R - right, L - left, MNI - Montreal Neurological Institute

and ALFF increased in the bilateral cerebellum posterior lobe, right precuneus, left middle orbitofrontal gyrus, and left medial superior frontal gyrus. The decrease of regional CBF was mainly observed in the left precentral gyrus and right middle frontal gyrus, and the increase of regional $\mathrm{CBF}$ in the bilateral superior parietal gyrus and right middle temporal gyrus. These altered brain regions were pain perception and modulation, such as sensory, motor, cognitive, and emotional functions, which might improve our understanding of the central pain processing mechanisms of CNSP from the perspective of neurovascular coupling.

\section{The default mode network}

Our study found decreased ALFF in the left precuneus, right cingulate gyrus, and left middle temporal gyrus, and increased ALFF in the right precuneus. We also found increased $\mathrm{CBF}$ in the right middle temporal gyrus. The precuneus, cingulate gyrus, and middle temporal gyrus are parts of the default mode network (DMN) [26]. The DMN is usually active during rest and is suspended or weakened when engaged with the external environment $[27,28]$. The DMN is generally the primary network affected by chronic pain [29]. Baliki et al. [30] found that the DMN was modulated and reorganised by the presence of chronic pain, which reflected the maladaptive physiology of different types of chronic pain. The precuneus played a major role in a lot of advanced cognitive functions, while the cingulate cortex was involved in cognitive, affective, and motor processing functions during empathy induction $[31,32]$. Jiang et al. [33] demonstrated that patients with postherpetic neuralgia showed decreased connectivity in the precuneus and posterior cingulate cortex of the DMN. Although the temporal lobe is considered to be one of the brain regions of pain integration, the temporal lobe is also involved in pain perception and modulation [34,35]. Tessitore et al. [36] found that patients with migraine without aura showed decreased connectivity in the prefrontal and temporal gyrus of the DMN. In our present work, abnormal ALFF 


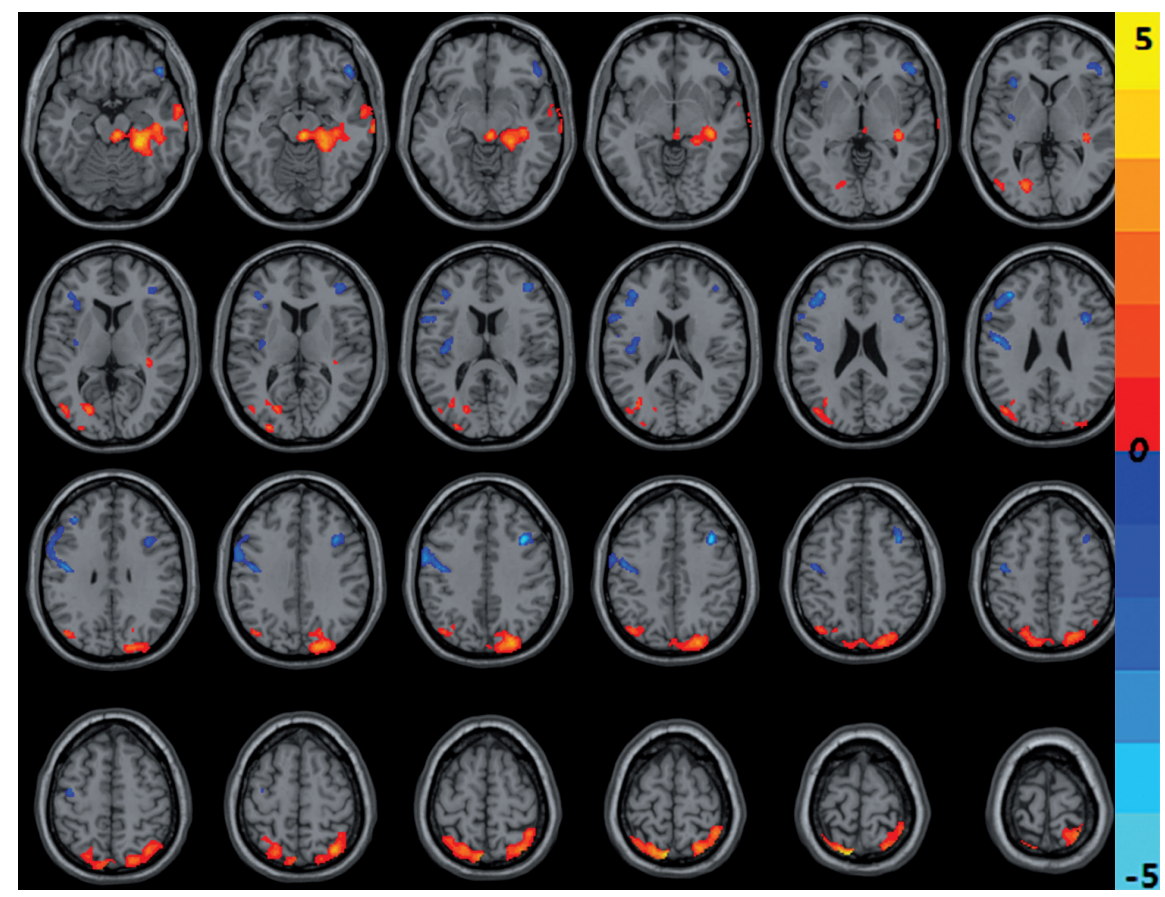

Figure 2. The cold and warm colours, respectively, indicated that the cerebral blood flow value decreased and increased in the brain regions in chronic neck and shoulder pain patients compared with healthy controls $(p<0.05$, AlphaSim corrected)

or $\mathrm{CBF}$ of the middle temporal gyrus, cingulate gyrus, and precuneus were detected in the brain scans. However, the altered coupling of FC and CBF between these regions within the $\mathrm{DMN}$ remain to be explored.

\section{Frontal regions}

In this study, the medial superior frontal gyrus and middle orbitofrontal gyrus showed increased ALFF, while the middle frontal gyrus and precentral gyrus displayed decreased ALFF. In addition, we also found that the right middle frontal gyrus of decreased ALFF was consistent with that of decreased CBF in CNSP patients. The prefrontal lobe is thought to play an important role in modulating the cortical and subcortical damaging pathways [37]. Besides pain processing, it was associated with depression and anxiety [38]. Mathew et al. [39] found that generalised anxiety disorder is associated with asymmetric increases in the $\mathrm{N}$-acetylaspartate/ creatine ratio, a suggested marker of neuronal viability in the prefrontal cortex. In this study, the ALFF value of the left medial superior frontal gyrus was positively correlated with VAS score. It indicated that the spontaneous nerve activity in this brain region can objectively reflect the pain intensity of CNSP patients, which may be a potential biomarker for clinical, objective evaluation of pain. The precentral gyrus is located in the frontal lobe of the cerebral cortex, which is the main somatic motor cortex [40]. A study on classical trigeminal neuralgia by Chen et al. [41] showed that the ALFF value of the right precentral gyrus was significantly higher in CTN patients when compared to HCs. Zhang et al. [21] found that elevated $\mathrm{CBF}$ value was observed in the right precentral gyrus of females with primary dysmenorrhea.
Table 3. Brain regions with cerebral blood flow differences between the chronic neck and shoulder pain and healthy controls groups

\begin{tabular}{|c|c|c|c|c|c|}
\hline \multirow[t]{2}{*}{ Region $(\mathrm{R} ; \mathrm{L})$} & \multirow{2}{*}{$\begin{array}{l}\text { Voxel } \\
\text { size }\end{array}$} & \multicolumn{3}{|c|}{ Peak MNI Coordinate } & \multirow{2}{*}{$\begin{array}{c}\text { Peak } \\
t \text {-value }\end{array}$} \\
\hline & & $X$ & $Y$ & Z & \\
\hline \multicolumn{6}{|l|}{ CNSP $<$ HCS } \\
\hline Precentral_L & 559 & -36 & 36 & 30 & -3.2527 \\
\hline Frontal_Mid_R & 271 & 36 & 16 & 42 & -3.7106 \\
\hline \multicolumn{6}{|l|}{$\mathrm{CNSP}>\mathrm{HCS}$} \\
\hline Parietal_Sup_L & 431 & -10 & -70 & 70 & 3.9 \\
\hline Parietal_Sup_R & 829 & -10 & -70 & 70 & 3.9 \\
\hline Temporal_Mid_R & 235 & 72 & -20 & -12 & 2.8171 \\
\hline
\end{tabular}

CNSP - chronic neck and shoulder pain, HCS - healthy controls, R - right, L - left, MNI - Montreal Neurological Institute

But in this study, we found that the precentral gyrus of decreased ALFF was consistent with that of decreased CBF in CNSP patients. Therefore, our findings suggest that frontal lobe dysfunction in the processing of pain or negative emotion may be related to the generating neural mechanism of CNSP.

\section{Parietal regions}

We found decreased ALFF in the right postcentral gyrus and increased CBF in the bilateral superior parietal gyrus. The postcentral gyrus, also known as the primary somatosensory cortex (S1), is located in the lateral parietal lobe of the brain, which contributes to the processing of tactile information, as well as the suppression of painful stimuli $[42,43]$. Previous studies have provided inconsistent findings for this region. A chronic pain study involved 


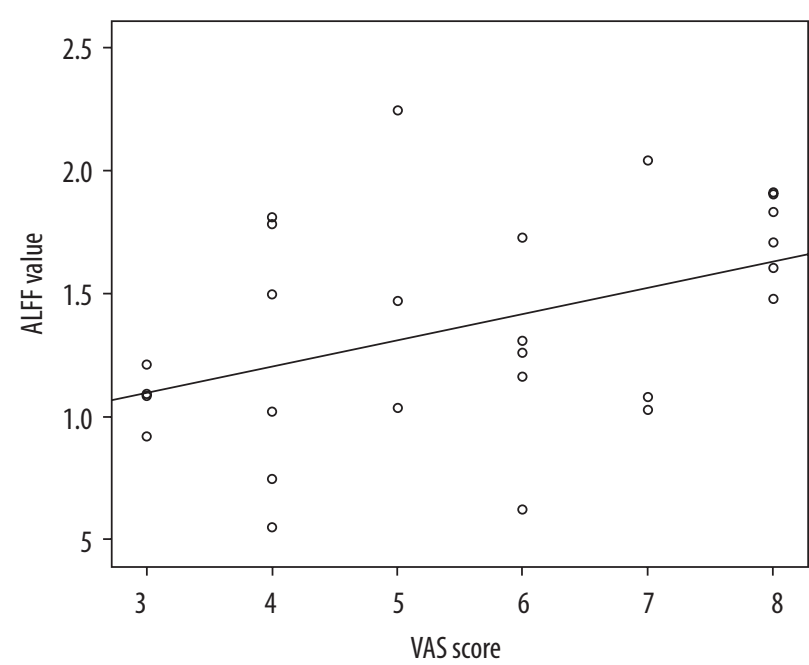

Figure 3. Correlation between the visual analogue scale (VAS) score and the amplitude of low-frequency fluctuation (ALFF) value in the left medial superior frontal gyrus $\left(R^{2}=0.182, p=0.023\right)$

increased brain connectivity to $S 1$ in patients with rheumatoid arthritis [44]. Another study found that patients with toothaches showed significantly higher ALFF in the left postcentral gyrus [45]. We can therefore speculate that the decreased ALFF value in the postcentral gyrus indicated activation of brain activities in S1, which affected pain perception. A recent study found that patients with chronic neck pain had increased volume in the left superior parietal gyrus compared to healthy controls [46]. This finding is consistent with the higher perfusion of bilateral superior parietal gyrus in this study.

\section{Occipital region}

We observed decreased ALFF in the right middle occipital gyrus of CNSP patients. The middle occipital gyrus, located in the extrastriatum of the visual cortex, is pertaining to visual communication. A study suggested that patients with blindness had changes in the thickness of the middle occipital gyrus [47]. Another study reported that patients with discogenic low back and leg pain showed increased ALFF in the left middle occipital gyrus [16]. However, our study had inconsistent findings for this region. So, the abnormal ALFF value of the middle occipital gyri in our study may have been related to this phenomenon, but the specific mechanism needs further study.

\section{Cerebellum}

In this study, the bilateral cerebellum posterior lobe of CNSP patients displayed higher ALFF values relative to HCs. The cerebellum was part of the pain matrix and always activated in painful events in healthy humans and in patients with chronic pain $[48,49]$. Besides pain modulation, the cerebellum was associated with all dimensions in regards to pain, including emotion, cognition, sensory function, and motor control [50,51]. Borsook et al. [52] conducted two studies on the effects of brush and heat stimuli applied to healthy and neuropathic pain subjects, so they proposed that the cerebellum may modulate the emotional and cognitive experience that distinguishes the perception of pain from the appreciation of innocuous sensory stimulation. Our study suggested that the bilateral cerebellum posterior lobe might participate in secondary sensory processing and cognition in patients with CNSP.

\section{Conclusions}

We combined the rs-fMRI and ASL MRI techniques to detect altered ALFF and CBF values in specific brain regions of CNSP patients, which mainly involved pain modulation and perception. This provides complementary information to reveal potential pathological mechanisms of CNSP. We also found that the ALFF value of the left medial superior frontal gyrus was positively correlated with the VAS score. The ALFF signals could be an effective marker to reflect pain severity.

\section{Limitations}

However, several limitations existed in the current study. First, the number of our cases was small, and we will recruit more patients to provide more precise results in the future. Second, there is no scale to evaluate the subjects' emotional states such as anxiety, depression, and so on, because different emotional state and severity may affect the brain functional network. Finally, we only observed the changes of ALFF and CBF in given brain regions in rs-fMRI. The altered coupling of FC and CBF should be included to reflect the neurovascular network integration in the follow-up study.

\section{Conflict of interest}

The authors declare no conflict of interest.

\section{References}

1. Chen B, Zhang C, Zhang RP, et al. Acupotomy versus acupuncture for cervical spondylotic radiculopathy: protocol of a systematic review and meta-analysis. BMJ Open 2019; 9: e29052.

2. Thoomes EJ, Scholten-Peeters GG, de Boer AJ, et al. Lack of uniform diagnostic criteria for cervical radiculopathy in conservative intervention studies: a systematic review. Eur Spine J 2012; 21 : 1459-1470.

3. Ohayon MM, Stingl JC. Prevalence and comorbidity of chronic pain in the German general population. J Psychiatr Res 2012; 46: 444-450. 
4. Kumbhare DA, Elzibak AH, Noseworthy MD. Evaluation of chronic pain using magnetic resonance (MR) neuroimaging approaches: what the clinician needs to know. Clin J Pain 2017; 33: 281-290.

5. Liu J, Gu L, Huang Q, et al. Altered gray matter volume in patients with herpes zoster and postherpetic neuralgia. J Pain Res 2019; 12: 605-616.

6. Chen C, Yan M, Yu Y, et al. Alterations in regional homogeneity assessed by fMRI in patients with migraine without aura. J Med Syst 2019; 43: 298.

7. Jung YH, Kim H, Jeon SY, et al. Neurometabolite changes in patients with complex regional pain syndrome using magnetic resonance spectroscopy: a pilot study. Neuroreport 2019; 30: 108-112.

8. Lee YC, Fine A, Protsenko E, et al. Brain correlates of continuous pain in rheumatoid arthritis as measured by pulsed arterial spin labeling. Arthritis Care Res (Hoboken) 2019; 71: 308-318.

9. Yu CX, Ji TT, Song H, et al. Abnormality of spontaneous brain activities in patients with chronic neck and shoulder pain: A resting-state fMRI study. J Int Med Res 2017; 45: 182-192.

10. Yu CX, Li B, Xu YK, et al. Altered functional connectivity of the periaqueductal gray in chronic neck and shoulder pain. Neuroreport 2017; 28: 720-725.

11. Yoshino A, Okamoto Y, Kunisato Y, et al. Distinctive spontaneous regional neural activity in patients with somatoform pain disorder: a preliminary resting-state fMRI study. Psychiatry Res 2014; 221: 246-248.

12. Napadow V, Harris RE. What has functional connectivity and chemical neuroimaging in fibromyalgia taught us about the mechanisms and management of 'centralized' pain? Arthritis Res Ther 2014; 16: 425.

13. Lee MJ, Park BY, Cho S, et al. Increased connectivity of pain matrix in chronic migraine: a resting-state functional MRI study. J Headache Pain 2019; 20: 29

14. Zhang B, Jung M, Tu Y, et al. Identifying brain regions associated with the neuropathology of chronic low back pain: a resting-state amplitude of low-frequency fluctuation study. Br J Anaesth 2019; 123: e303-e311.

15. Chen Y, Xiang CQ, Liu WF, et al. Application of amplitude of low frequency fluctuation to altered spontaneous neuronal activity in classical trigeminal neuralgia patients: a restingstate functional MRI study. Mol Med Rep 2019; 20: 1707-1715.

16. Zhou F, Gu L, Hong S, et al. Altered low-frequency oscillation amplitude of resting state-fMRI in patients with discogenic low-back and leg pain. J Pain Res 2018; 11: 165-176.

17. Barkhof F, Haller S, Rombouts SA. Resting-state functional MR imaging: a new window to the brain. Radiology 2014; 272: 29-49.

18. Zhang C, Dou B, Wang J, et al. Dynamic alterations of spontaneous neural activity in Parkinson's disease: a resting-state fMRI study. Front Neurol 2019; 10: 1052.

19. Zhu J, Zhuo C, Xu L, et al. Altered coupling between resting-state cerebral blood flow and functional connectivity in schizophrenia. Schizophr Bull 2017; 43: 1363-1374.

20. Liang X, Zou Q, He Y, Yang Y. Coupling of functional connectivity and regional cerebral blood flow reveals a physiological basis for network hubs of the human brain. Proc Natl Acad Sci U S A 2013; 110: 1929-1934.
21. Zhang YN, Huo JW, Huang YR, et al. Altered amplitude of lowfrequency fluctuation and regional cerebral blood flow in females with primary dysmenorrhea: a resting-state fMRI and arterial spin labeling study. J Pain Res 2019; 12: 1243-1250.

22. Alsop DC, Detre JA, Golay X, et al. Recommended implementation of arterial spin-labeled perfusion MRI for clinical applications: a consensus of the ISMRM perfusion study group and the European consortium for ASL in dementia. Magn Reson Med 2015; 73: 102-116.

23. Zhou F, Huang M, Wu L, et al. Altered perfusion of the sensorimotor cortex in patients with cervical spondylotic myelopathy: an arterial spin labeling study. J Pain Res 2018; 11: 181-190.

24. Yu H, Qiu X, Zhang YQ, et al. Abnormal amplitude of low frequency fluctuation and functional connectivity in non-neuropsychiatric systemic lupus erythematosus: a resting-state fMRI study. Neuroradiology 2019; 61: 331-340.

25. Zang YF, He Y, Zhu CZ, et al. Altered baseline brain activity in children with ADHD revealed by resting-state functional MRI. Brain Dev 2007; 29: 83-91.

26. Raichle ME. The brain's default mode network. Annu Rev Neurosci 2015; 38: 433-447.

27. Wang C, Pan Y, Liu Y, et al. Aberrant default mode network in amnestic mild cognitive impairment: a meta-analysis of independent component analysis studies. Neurol Sci 2018; 39: 919-931.

28. Zuo N, Yang Z, Liu Y, et al. Core networks and their reconfiguration patterns across cognitive loads. Hum Brain Mapp 2018; 39: 35463557.

29. Farmer MA, Baliki MN, Apkarian AV. A dynamic network perspective of chronic pain. Neurosci Lett 2012; 520: 197-203.

30. Baliki MN, Mansour AR, Baria AT, Apkarian AV. Functional reorganization of the default mode network across chronic pain conditions. PLoS One 2014; 9: e106133.

31. Cavanna AE, Trimble MR. The precuneus: a review of its functional anatomy and behavioural correlates. Brain 2006; 129 (Pt 3): 564-583.

32. Yesudas EH, Lee TM. The role of cingulate cortex in vicarious pain. Biomed Res Int 2015; 2015: 719615.

33. Jiang J, Gu L, Bao D, et al. Altered homotopic connectivity in postherpetic neuralgia: a resting state fMRI study. J Pain Res 2016; 9: 877-886.

34. Cao S, Qin B, Zhang Y, et al. Herpes zoster chronification to postherpetic neuralgia induces brain activity and grey matter volume change. Am J Transl Res 2018; 10: 184-199.

35. Ploner M, Lee MC, Wiech K, et al. Flexible cerebral connectivity patterns subserve contextual modulations of pain. Cereb Cortex 2011; 21: 719-726.

36. Tessitore A, Russo A, Giordano A, et al. Disrupted default mode network connectivity in migraine without aura. J Headache Pain 2013; 14: 89.

37. Yang FC, Chou KH, Fuh JL, et al. Altered gray matter volume in the frontal pain modulation network in patients with cluster headache. Pain 2013; 154: 801-807.

38. Vialou V, Bagot RC, Cahill ME, et al. Prefrontal cortical circuit for depression- and anxiety-related behaviors mediated by cholecystokinin: role of DeltaFosB. J Neurosci 2014; 34: 3878-3887.

39. Mathew SJ, Mao X, Coplan JD, et al. Dorsolateral prefrontal cortical pathology in generalized anxiety disorder: a proton magnetic 
resonance spectroscopic imaging study. Am J Psychiatry 2004; 161 1119-1121.

40. Yousry TA, Schmid UD, Alkadhi H, et al. Localization of the motor hand area to a knob on the precentral gyrus. A new landmark. Brain 1997; 120 (Pt 1): 141-157.

41. Chen Y, Xiang CQ, Liu WF, et al. Application of amplitude of low frequency fluctuation to altered spontaneous neuronal activity in classical trigeminal neuralgia patients: a restingstate functional MRI study. Mol Med Rep 2019; 20: 1707-1715.

42. Ploner M, Schmitz F, Freund HJ, Schnitzler A. Differential organization of touch and pain in human primary somatosensory cortex. J Neurophysiol 2000; 83: 1770-1776.

43. Vartiainen N, Kirveskari E, Kallio-Laine K, et al. Cortical reorganization in primary somatosensory cortex in patients with unilateral chronic pain. J Pain 2009; 10: 854-859.

44. Flodin P, Martinsen S, Altawil R, et al. Intrinsic brain connectivity in chronic pain: a resting-state fMRI study in patients with rheumatoid arthritis. Front Hum Neurosci 2016; 10: 107.

45. Yang J, Li B, Yu QY, et al. Altered intrinsic brain activity in patients with toothaches using the amplitude of low-frequency fluctuations: a resting-state fMRI study. Neuropsychiatr Dis Treat 2019; 15: 283-291.

46. De Pauw R, Coppieters I, Caeyenberghs K, et al. Associations between brain morphology and motor performance in chronic neck pain: a whole-brain surface-based morphometry approach. Hum Brain Mapp 2019; 40: 4266-4278.

47. Anurova I, Renier LA, De Volder AG, et al. Relationship between cortical thickness and functional activation in the early blind. Cereb Cortex 2015; 25: 2035-2048.

48. Helmchen C, Mohr C, Erdmann C, et al. Differential cerebellar activation related to perceived pain intensity during noxious thermal stimulation in humans: a functional magnetic resonance imaging study. Neurosci Lett 2003; 335: 202-206.

49. Jensen KB, Regenbogen C, Ohse MC, et al. Brain activations during pain: a neuroimaging meta-analysis of patients with pain and healthy controls. Pain 2016; 157: 1279-1286.

50. Moulton EA, Schmahmann JD, Becerra L, Borsook D. The cerebellum and pain: passive integrator or active participator? Brain Res Rev 2010; 65: 14-27.

51. Allen G, McColl R, Barnard H, et al. Magnetic resonance imaging of cerebellar-prefrontal and cerebellar-parietal functional connectivity. Neuroimage 2005; 28: 39-48.

52. Borsook D, Moulton EA, Tully S, et al. Human cerebellar responses to brush and heat stimuli in healthy and neuropathic pain subjects. Cerebellum 2008; 7: 252-272. 